\title{
Transverse vs torsional ultrasound: prospective randomized contralaterally controlled study comparing two phacoemulsification-system handpieces
}

\author{
This article was published in the following Dove Press journal: \\ Clinical Ophthalmology \\ 3 August 2015 \\ Number of times this article has been viewed
}

\section{Kerry K Assil \\ Lindsay Harris \\ Jeannie Cecka}

Assil Eye Institute, Beverly Hills, CA, USA
Correspondence: Kerry K Assil Assil Eye Institute, 3rd floor, 450 North Roxbury Drive, Beverly Hills, CA 90210 , USA

Tel +I 310 65I 2300

Email kerry@assileye.com
Purpose: To compare surgical efficiency and multiple early clinical outcome variables in eyes undergoing phacoemulsification using either transversal or torsional ultrasound systems.

Setting: Assil Eye Institute, Beverly Hills, CA, USA.

Design: Prospective, randomized, clinician-masked, contralaterally controlled single-center evaluation.

Patients and methods: Patients seeking cataract removal in both eyes with implantation of multifocal intraocular lenses were randomly assigned to one of two treatment rooms for phacoemulsification with either a transverse ultrasound system or torsional handpiece system. The contralateral eye was treated at a later date with the alternate device. A total of 54 eyes of 27 patients having similar degrees of cataract, astigmatism, and visual potential were included. All operative data were collected for analysis, and patients were followed for 3 months after surgery.

Results: Similar visual acuity was reported at all postoperative visits between the two groups. Mean phacoemulsification time and total power required were both significantly lower with the transverse system than with the torsional technique $(P<0.05$ for both). Similarly, mean total balanced salt solution used was significantly less with the transverse system vs torsional $(P<0.05)$. Postoperative safety demonstrated significantly lower endothelial cell loss at 1 day and 1 month $(P<0.05)$ with transverse vs torsional. Macular swelling was less at 1 week, 1 month, and 3 months with transverse vs torsional, although the difference did not achieve significance $(P=0.1)$ at any single time point. Clinically detectable corneal edema was reported less frequently at all postoperative time points with the transverse system.

Conclusion: The transverse ultrasound system was found to be possibly associated with less balanced salt-solution use, less phacoemulsification time, and less power required than the torsional phaco system. Postoperative data suggested that improved phaco efficiency may translate to a better overall safety profile for the patient.

Keywords: phacoemulsification, transversal, torsional, cutting efficiency

\section{Introduction}

Over 20 million Americans 40 years of age or older are affected by cataracts. Removal of the cataract by phacoemulsification, together with implantation of an intraocular lens (IOL), continues to be the most commonly performed surgical procedure in the USA, with the number of cataract procedures performed each year having grown to over $1,500,000 .^{1}$ 
Improvements in surgical technique, phacoemulsification technology, viscoelastic substances, and IOLs now enable over $97 \%$ of patients to recover from cataract surgery with no major complications. ${ }^{2}$ Despite these promising results, enhanced efficiency and safety remain the top priority for continued research and product development. Surgeons continue to seek advances in phacoemulsification cutting efficiency, reduced time, and lower power settings, in order to further prevent complications, such as incision burn, endothelial cell loss, and pseudophakic cystoid macular edema. ${ }^{3,4}$ Among the technological innovations that are undergoing research and development in cataract extraction, overall reduction of phacoemulsification energy and time continue to be major objectives for future improvements. ${ }^{5}$ Research and development to continue to improve all aspects of phacoemulsification equipment and procedure have resulted in enhancements of vacuum and flow settings, reduction in the amount of energy delivered, and reduced phacoemulsification time. ${ }^{6,7}$

With conventional phacoemulsification, the ultrasound power used to emulsify the lens originates from the longitudinal movement of the phacoemulsification needle, with the tip of the handpiece moving at a high frequency in an advancing and retreating motion. ${ }^{8}$ The ultrasound mode can produce a repulsion effect, because the phaco tip pushes the nucleus away with each stroke as it advances. As a result, the ultrasonic impact upon the lens fragments can be interrupted and efficacy compromised. Transverse ultrasound-power modulation is a technological development that minimizes chatter from the nuclear fragments that are being emulsified. ${ }^{9}$ This type of modulation results in the ability of a "side-toside" movement that may reduce the frictional heat generation noted with traditional longitudinal-only movement. The torsional oscillation effect is another developmental improvement that is noted to reduce the amount of phacoemulsification energy and increase the efficiency required to remove the cataractous nucleus by fragmenting the cataract via shearing, in place of the conventional jackhammer effect. ${ }^{9}$ These developments in oscillation, together with the advancement of micropulse-energy delivery, have resulted in reduced repulsion of the lens material during aspiration and improved "followability", which is the beneficial tendency of nuclear fragments to be aspirated in a relatively feathery and continuous fashion. ${ }^{6}$

Two of the newer phacoemulsification systems incorporating these advances are a transverse ultrasound system (WhiteStar Signature ${ }^{\mathrm{TM}}$ system with Ellips ${ }^{\circledR}$ FX Transversal Ultrasound; Abbott Medical Optics Inc, Santa Ana, CA,
USA) and a torsional system (Infiniti with the Ozil ${ }^{\circledR}$ torsional handpiece; Alcon Laboratories Inc, Fort Worth, TX, USA). The Infiniti Ozil uses torsional tip technology integrated into the Infiniti phacoemulsification system, producing an oscillatory movement along the longitudinal axis of the tip (eg, side-to-side movement), as opposed to the to-and-fro movement of traditional phaco tips. Torsional movement keeps the tip in contact with the cataract, reducing the chance of nuclear material repelling from the tip and decreasing heat production. ${ }^{4}$ With side-to-side torsional movement, emulsification is optimized when using a bent phaco tip. The WhiteStar Signature Ellips FX uses a combination of longitudinal and transversal phaco, which can be delivered with either a straight or bent phaco tip. Micropulses of power are delivered, generating side-to-side or longitudinal movement. The system also incorporates both peristaltic and Venturi fluidics. The fusion of different technologies into one system is purported to offer flexibility for the surgeon and possibly to improve efficiency.

A prospective, randomized study was conducted comparing the two phaco systems - the WhiteStar Signature system with Ellips FX transverse ultrasound handpiece and the Infiniti with Ozil torsional handpiece - to evaluate and compare intraoperative efficiency and postoperative outcomes, primarily with regard to visual acuity, ocular (corneal and macular) edema, and inflammation. The outcome variables were measured preoperatively and postoperatively.

\section{Patients and methods}

This single-site study was designed as a prospective, clinicianmasked, contralaterally controlled evaluation of 54 eyes of 27 patients. For their first eye, patients were randomly assigned to one of two treatment rooms to undergo bilateral phacoemulsification with implantation of a multifocal IOL, according to a randomization schedule. One treatment room was equipped with the Ellips FX transverse system, and the second room was equipped with the Infiniti with Ozil torsional system. Table 1 presents the specific settings used by the surgeon on each phacoemulsification machine with regard to sculpt, chop, and epinucleus parameters. Within 6 weeks following the first eye surgery, the contralateral (second) eye underwent cataract surgery using the alternate phacoemulsification device. Patients in the study were implanted with either the Sensar ${ }^{\circledR}$ monofocal IOL with OptiEdge ${ }^{\text {TM }}$ or the Tecnis ${ }^{\circledR}$ multifocal IOL (both Abbott Medical Optics). Patients received the same IOL in both eyes. The investigator and surgeon (KA) is an established board-certified ophthalmologist who is experienced in using both phacoemulsification systems 
Table I Phaco-system settings

\begin{tabular}{|c|c|c|}
\hline Machine settings & $\begin{array}{l}\text { AMO WhiteStar Signature } \\
\text { phaco system }\end{array}$ & $\begin{array}{l}\text { Alcon Infinity with Ozil } \\
\text { phaco system }\end{array}$ \\
\hline \multirow[t]{4}{*}{ Sculpt } & Aspiration: $22 \mathrm{cc} / \mathrm{min}$ & Aspiration: $27 \mathrm{cc} / \mathrm{min}$ \\
\hline & Vacuum: 40 mmHg (peristaltic) & Vacuum: 150 mmHg (peristaltic) \\
\hline & Power: 50\% (transverse/longitudinal) & Power: $20 \%$ (torsional amplitude) \\
\hline & $65 \mathrm{~cm} / \mathrm{H}_{2} \mathrm{O}$ (bottle height) & $95 \mathrm{~cm} / \mathrm{H}_{2} \mathrm{O}$ (bottle height) \\
\hline \multirow[t]{4}{*}{ Chop } & Vacuum: $250 \mathrm{mmHg}$ (Venturi) & Vacuum: $320 \mathrm{mmHg}$ (peristaltic) \\
\hline & Power: $40 \%$ & Aspiration: $32 \mathrm{cc} / \mathrm{min}$ \\
\hline & $70 \mathrm{~cm} / \mathrm{H}_{2} \mathrm{O}$ (bottle height) & Power: $60 \%$ (torsional amplitude) \\
\hline & & $95 \mathrm{~cm} / \mathrm{H}_{2} \mathrm{O}$ (bottle height) \\
\hline \multirow[t]{4}{*}{ Epinucleus } & Aspiration: $38 \mathrm{cc} / \mathrm{min}$ & Aspiration: $30 \mathrm{cc} / \mathrm{min}$ \\
\hline & Vacuum: $350 \mathrm{mmHg}$ (peristaltic) & Vacuum: $230 \mathrm{mmHg}$ (peristaltic) \\
\hline & Power: $20 \%$ & Power: $60 \%$ \\
\hline & $60 \mathrm{~cm} / \mathrm{H}_{2} \mathrm{O}$ (bottle height) & $95 \mathrm{~cm} / \mathrm{H}_{2} \mathrm{O}$ (bottle height) \\
\hline
\end{tabular}

Abbreviation: AMO, Abbott Medical Optics.

and handpieces. KA had used the phaco systems interchangeably for the 6 months prior to initiation of the study, in order to optimize the settings for each system.

Patients were enrolled in the trial if they were at least 21 years of age, anticipated to undergo phacoemulsification in both eyes with IOL implantation, and were willing, available, and of sufficient cognitive awareness to comply with the examination procedures. Patients were offered the option of a monofocal or multifocal IOL, and were informed that the same IOL would be implanted in each eye. Both eyes were required to have similar degrees of cataract and astigmatism, including topographic cylinder difference of less than $2.0 \mathrm{D}$ and globe axial length difference of less than $0.5 \mathrm{~mm}$, as measured by the IOLMaster ${ }^{\circledR}$ (Carl Zeiss Meditec AG, Jena, Germany). Patients were excluded if the anticipated visual potential of either eye was worse than 20/25, were undergoing asymmetrical use of ocular medications, or were noted to have the presence of glaucoma, macular disease, or corneal disease in either eye.

Following institutional review board approval and acceptance of the written informed consent by each subject, patients underwent a standard preoperative exam. All steps in this study were conducted in an ethical manner in accordance with the Declaration of Helsinki. Manifest refraction, visual acuity, and grading of the cataract (according to the nuclear color and opalescence grades of the Lens Opacities Classification System III) was carried out and recorded. Also, the standard tests of intraocular pressure, biometry, and slit-lamp evaluation were performed. In order to assess comparative outcomes in both randomized groups, additional testing was performed, including corneal pachymetry (Orbscan II scanning slit topography; Bausch \& Lomb Inc, Bridgewater, NJ, USA), specular microscopy (CellChek;
Konan Medical Inc, Nishinomiya, Japan) and assessment of macular thickness with optical coherence tomography (OCT; Cirrus high-definition SD-OCT; Carl Zeiss). All of these tests were performed at the preoperative and postoperative visits.

Phacoemulsification and implantation of the IOL was performed using the surgeon's standard procedure, including a perilimbal clear corneal temporal incision measuring $2.5 \mathrm{~mm}$. The surgeon performed central grading with horizontal nuclear chopping using a bimanual technique, followed by coaxial, irrigation/aspiration, and capsular polishing. Operative outcome measures included notation of total phacoemulsification time, total volume of balanced salt solution (BSS; Alcon) used, time required for hydration-technique wound closure, and phacoemulsification-power settings. The occurrence of any operative complications or adverse events was also noted.

Postoperatively, patients returned for evaluation at 1 day, 1 week, 1 month, and 3 months. At these visits, measurements included uncorrected visual acuity (LogMAR), specular microscopy, corneal pachymetry, intraocular pressure, and macular OCT. A comprehensive slit-lamp exam was also performed at all visits to grade corneal epithelial punctate staining and degree of stromal edema; edema was graded using a 5-point scale. The clinical evaluator was trained by the investigator for consistency, and was masked with respect to the specific phacoemulsification device used. At the 3-month visit, mandatory measurements consisted only of specular microscopy and macular thickness. Adverse postoperative events were defined as any untoward medical occurrence that did or did not necessarily have a causal relationship with this treatment, including a worsening of any previously identified condition. 
Analyses of continuous variables were performed with either parametric or nonparametric tests as appropriate. Nominal variables were compared with the $\chi^{2}$ or Fisher's exact tests. The a priori level of significance was $\alpha=0.05$ for all tests. $P$-values were calculated by means of a two-sided paired $t$-test of the difference in means. Results were deemed statistically significant if $P<0.05$. Statistical analysis was performed using SAS software (SAS Institute Inc, Cary, NC, USA).

\section{Results}

\section{Patient demographics}

Cataract surgery was performed by a single surgeon (KA) on 54 eyes of 27 patients between July and September 2011. Baseline characteristics between the two randomly assigned groups (Ellips FX and Infiniti Ozil) were comparable. The mean age of the patient population at the time of surgery was 69 years \pm 7.8 (standard deviation); $65 \%$ of patients were female. Mean preoperative manifest refraction spherical equivalent for the overall population was $-0.16 \mathrm{D}$. All patients completed the prospective study follow-up.

\section{Operative outcomes}

The surgeon's standard phacoemulsification technique was performed in all cases in both the Ellips FX and Infiniti Ozil groups. With regard to surgical efficiency, the effective phaco time for Ellips FX was 31.97 seconds shorter than with Infiniti Ozil, and was statistically significant $(P=0.0019)$. The confidence interval $(-50.43$ to -13.52$)$ indicated that at best, Ozil took 13.52 seconds longer than Ellips, and at worst, effective phaco time was 50.43 seconds longer with Ozil than Ellips. The mean required phacoemulsification-power setting was lower with the Ellips FX system (45.2 mJ) than the Infiniti Ozil (62.5 mJ; $P=0.208$ ) (Figure 1). The mean difference in power was $14.17 \mathrm{~mJ}$ (higher for Infiniti), which was not statistically significant.

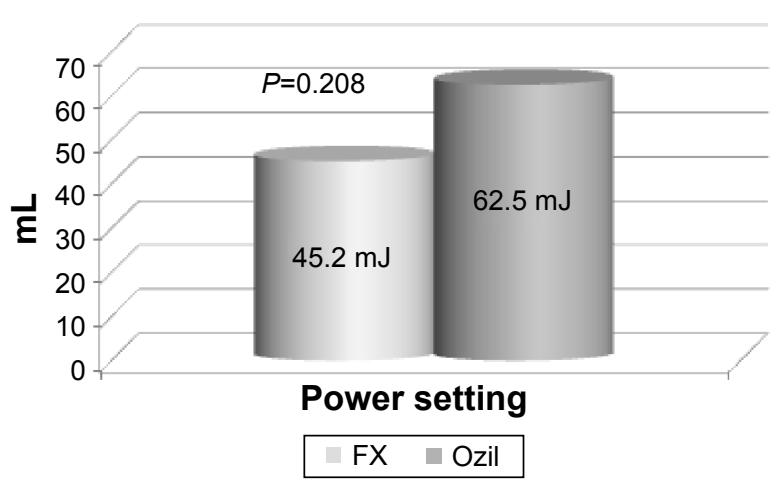

Figure I Phaco-power setting FX vs Ozil.
The mean volume of BSS used during surgery was significantly less with the Ellips FX $(313 \mathrm{cc})$ than the Infiniti Ozil handpiece (350 cc) $(P=0.037)$ (Figure 2). Mean time for hydration-technique wound closure was slightly lower with the Ellips FX handpiece (15.4 seconds) vs the Infiniti Ozil handpiece (16.1 seconds; $P>0.05$ ).

No surgical adverse events (including corneal burns, detached Descemet's membrane, iris trauma, intraocular bleeding, or capsular ruptures) were observed with either instrument. No patients required operative medications outside the surgeon's standard regimen.

\section{Postoperative outcomes}

Statistically significant differences in postoperative findings were noted at various visits between the two treatment groups for endothelial cell loss and change in corneal pachymetry. Postoperative endothelial cell loss was significantly less at both the 1-day and 1-month follow-up periods in the Ellips FX group. In the FX group, the mean reduction in endothelial cells measured 45.1 cells at 1 day and 127.3 cells at 1 month, compared to Ozil, in which the reduced cell counts measured 170.7 at 1 day and 382.3 at 1 month $(P<0.05$ for both time points). This difference became diminished, and did not achieve significance at 3 months. (Figure 3).

The mean change in corneal pachymetry for each visit during the 3-month follow-up is illustrated in Figure 4. The change in corneal thickness at 1 day postsurgery was statistically significantly less with the Ellips FX $(P=0.046)$ than the Infiniti Ozil. At day 1 with FX, mean change in corneal pachymetry was $30.3 \mu \mathrm{m}$ vs $51.0 \mu \mathrm{m}$ with Ozil.

Slit-lamp exam observations confirmed less frequent corneal stromal edema in the Ellips FX group at postoperative day 1; no stromal edema was noted in $77.7 \%$ of Ellips FX eyes compared to only $30.3 \%$ of Infiniti Ozil eyes. No moderate or severe corneal edema was seen in the Ellips

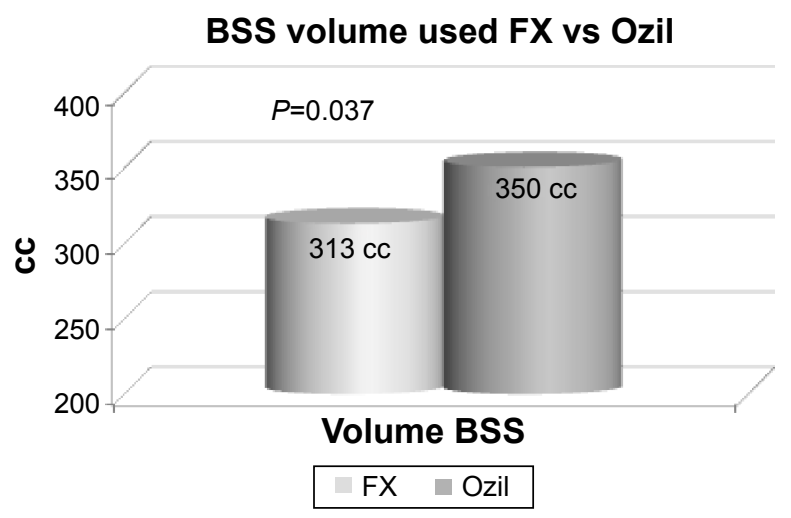

Figure 2 Mean volume balanced salt solution (BSS) used. 


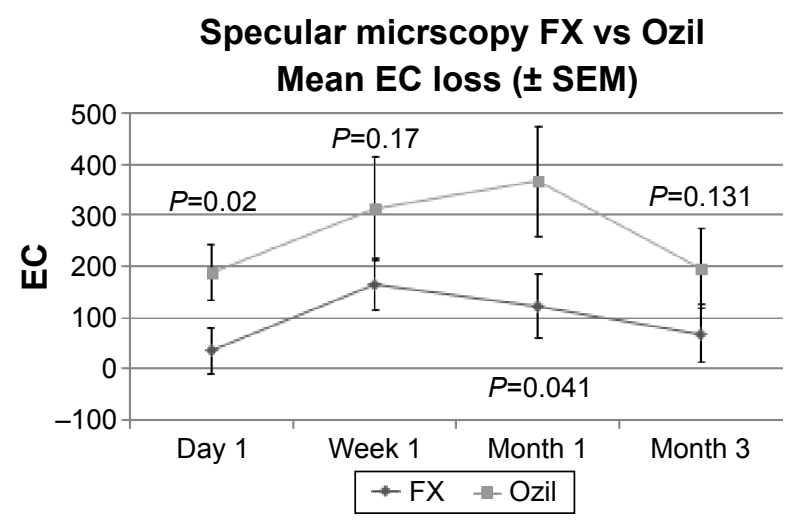

Figure 3 Mean endothelial cell (EC) loss throughout postoperative period. Abbreviation: SEM, standard error of mean.

FX eyes compared to the Infiniti Ozil group, with three eyes (11\%) having moderate or severe corneal edema reported (Figure 5). No corneal stromal edema was observed in either group at 1 week or later after surgery.

Macular swelling as measured by mean change in OCTmeasured central macular thickness post-cataract removal was observed to be less in the Ellips FX group compared to the Infiniti Ozil group. This data only demonstrated a significant difference at the 1-week time point (Figure 6). However, when all time points were pooled, this also resulted in a significant difference between the two groups. The difference in mean pooled macular edema between the two groups was $-17.70(P=0.016)$. No postoperative adverse events were reported in any eyes enrolled in the study at any time during the follow-up period.

Uncorrected visual acuity (LogMAR) outcomes were similar between the two groups at all postoperative visits. There was no significant difference in uncorrected visual acuity between the Ellips FX- treated or Infiniti Ozil-treated

\section{Corneal pachymetry FX vs Ozil}
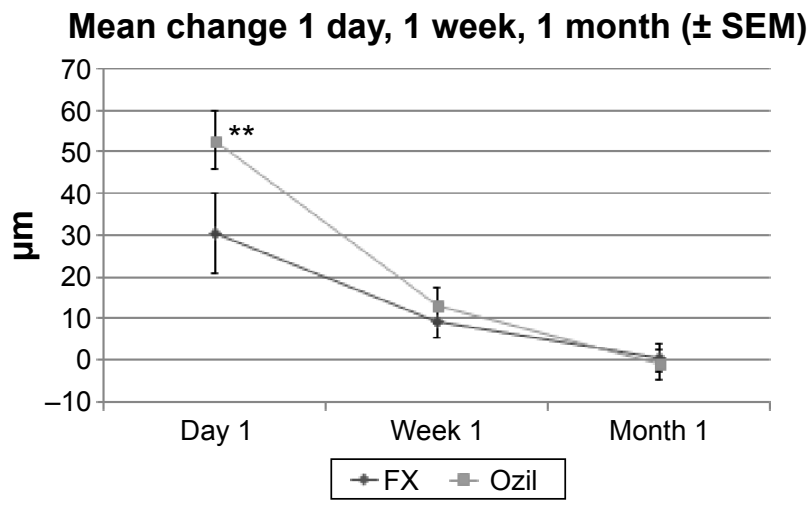

Figure 4 Mean change in corneal pachymetry throughout postoperative period. Note: ${ }^{* *} P<0.05$.

Abbreviation: SEM, standard error of mean.

\section{Corneal edema FX vs Ozil \\ 1 day postoperative}

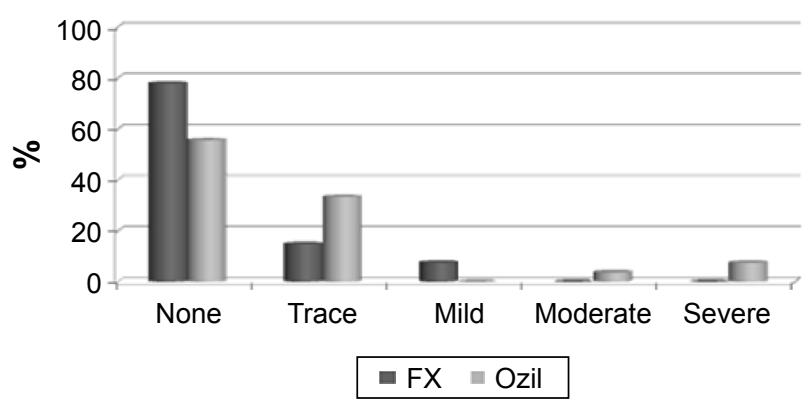

Figure 5 Corneal edema at I day postoperatively (PO).

Note: No eyes had corneal edema I week PO.

groups at any follow-up visit throughout the 3 -month period (Figure 7).

At the conclusion of each surgical procedure, the surgeon completed a standardized questionnaire to assess overall cutting efficiency, chamber stability, clogging of the phaco tip, and overall satisfaction with the performance of the handpiece and phaco machine used. In general, the analysis of the questionnaire data indicated similar outcomes with regard to handpiece performance and satisfaction with the equipment. The surgeon noted, however, that in two cases with the Infiniti Ozil, the tip clogged during the procedure, whereas there were no instances of clogging found with the Ellips FX.

\section{Discussion}

Numerous studies have confirmed that advances in phacoemulsification technology in recent years have ultimately provided a reduction in overall ultrasound energy used and time needed to remove the cataract, translating into improved efficiency and safety for the patient. We conducted

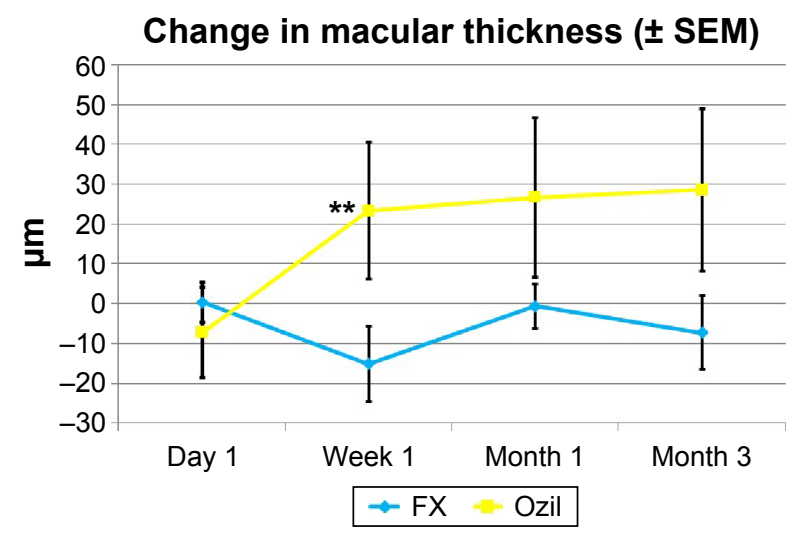

Figure 6 Macular thickness results.

Note: $* * P<0.05$.

Abbreviation: SEM, standard error of mean. 


\section{Postoperative UCVA FX vs Ozil}

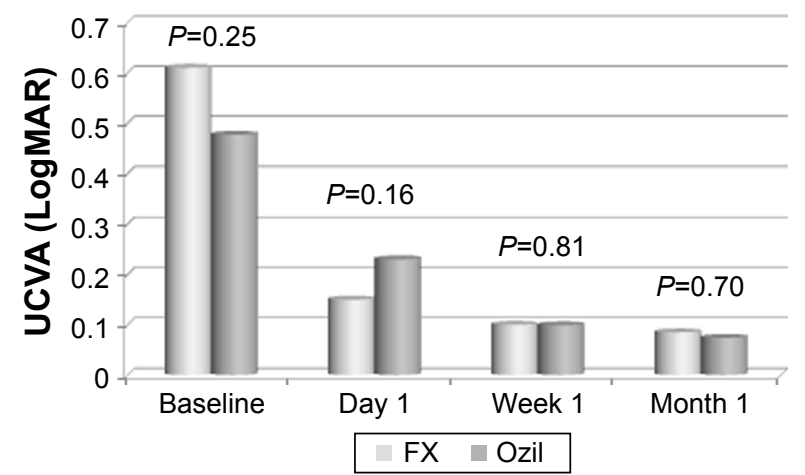

Figure 7 Postoperative uncorrected visual acuity (UCVA) throughout the postoperative period.

a prospective, randomized, observer-masked, single-site study to compare outcomes of two of the advanced, newtechnology phaco machines: the Infiniti with Ozil handpiece and the WhiteStar Signature with Ellips FX handpiece. Infiniti Ozil utilizes a torsional handpiece, with a side-toside, nonlongitudinal movement of the phaco tip. The Ellips FX handpiece blends two movements, incorporating both lateral and longitudinal components. The evaluation of these two devices was undertaken via a randomized, match-paired clinical randomized controlled trial, as this approach is believed to minimize allocation bias in the assignment of treatments. In addition to randomization of patients' eyes into one of two treatment groups, we sought to diminish outcome bias further by having all treatments performed by the same surgeon, utilizing corresponding equipment in each eye, conducting postoperative examinations utilizing uniform testing, and performing patient examinations by a single masked observer. The match-paired approach of comparing the two eyes of the same patient was further intended to minimize the impacts of demographic and interindividual variability.

Results demonstrated excellent intraoperative safety, efficiency, and overall performance with both systems. The transverse Ellips FX handpiece group, however, demonstrated a significantly lower phaco time $(P<0.05)$ and significantly less usage of BSS $(P<0.05)$ during the procedure vs the Infiniti Ozil. This may represent a clinically significant difference, as it has previously been reported that the single most important predictor of good vision and a clear cornea postoperatively is the total amount of phaco energy delivered into the eye. ${ }^{6}$ The benefit of less BSS usage during the procedure may suggest better surgeon control of the handpiece, better control of the nuclear fragments, and may also translate into a clinical reduction of corneal edema.
Clinically observable corneal edema had resolved in all patients in both groups by the 1 -week visit, although a difference in endothelial cell loss (favoring Ellips FX) was still observed at 3 months. Less phaco time and power may also correlate with the difference in corneal thickness measurement by corneal pachymetry at 1 day, which was statistically significantly less with the Ellips FX, thus possibly suggesting a more rapid early postoperative recovery.

Reducing endothelial trauma is also an anticipated positive outcome of less phaco energy expended during cataract extraction. Minimizing endothelial damage is important during phaco treatment, as iatrogenic cell loss may occur with excessive intraocular manipulation, ultrasonic vibration, and heat generated by the phaco tip. ${ }^{10}$ In our study, postoperative endothelial cell loss was less at all follow-up time points, and statistically significantly less at both the 1-day and 1-month follow-up periods in the Ellips FX group vs Infiniti Ozil. These findings may suggest a correlation between the statistically significant lower phaco time and power setting noted with the Ellips FX vs Infiniti Ozil and the consequent differences in endothelial cell loss.

The study also reported findings of less macular swelling with FX than with Ozil at 1 week, 1 month, and 3 months. Macular edema as measured by mean change in OCT central macular thickness was reported to be less in the Ellips FX group at all postoperative visits. A larger study with longer follow-up might determine if these reduced macular edema findings would translate into clinically significant outcome differences, such as reduction of early cystoid macular edema or the reduction of subsequent epiretinal membrane formation in this group of patients.

While the focus of this study was to evaluate the outcomes as a function of different handpieces, it is possible that the difference in endothelial cell loss and macular edema between the two groups can be explained by other factors. We note that the differential bottle height, aspiration, and vacuum settings needed to optimize chamber stability between the two groups may also have had an effect on outcomes. These differences may have led to differential intraocular pressure fluctuation and fluidics with associated turbulence, which may have influenced intraocular dynamics, resulting in endothelial cell loss or macular swelling.

Two cases in which Ozil was used were noted to have "clogging" of the phaco tip during removal of the cataract. Clogging of the tip may lead to the concern of heat buildup in and around the area of removal. This was further noted by Schmutz and Olson, who described that metal stress in the proximal phaco needle shaft can create substantial heat 
buildup with transverse ultrasound, especially with Ozil. ${ }^{11}$ In their paper, Ellips was found to be significantly less hot than Ozil with all test scenarios, especially in the clinically relevant scenario where the tip is occluded and there is no or little cooling flow through the tip.

\section{Conclusion}

In summary, this prospective, match-paired randomized, observer-masked study of two of the newer, advanced phacoemulsification machines available demonstrated excellent operative efficiency, with patients experiencing fast visual rehabilitation with no surgical or postoperative complications. The Ellips FX transverse ultrasound system allowed for a faster, possibly more efficient surgical procedure, with less BSS, less phacoemulsification time, and less power required than the Infiniti with the Ozil torsional handpiece. The improved surgical efficiency noted with FX may translate into improved safety, and is supported by the observed postoperative results in this study noting less corneal edema, less endothelial cell loss, and less macular edema.

A limitation of this study was the small subject population. A larger, multicenter study may be beneficial in providing greater correlation between enhanced surgical efficiency and postoperative recovery and safety.

\section{Acknowledgment}

Funding of the clinical study included a grant from Abbott Medical Optics.

\section{Author contributions}

KKA designed and conducted the study; collection, management, analysis, and interpretation of the data were carried out by KKA and LH; and preparation, review, or approval of the manuscript was undertaken by KKA and JC. All authors contributed toward data analysis, drafting and revising the paper and agree to be accountable for all aspects of the work.

\section{Disclosure}

KKA and LH were consultants to Abbott Medical Optics at the time the study was performed. The authors report no other conflict of interest in this work.

\section{References}

1. Cullen KA, Hall MJ, Golosinskiy A. Ambulatory surgery in the United States, 2006. Natl Health Stat Report. 2009;(11):1-25.

2. Feldman BH, Heersink S. Cataract. 2014. Available from: http://eyewiki.aao.org/Cataract. Accessed June 17, 2015.

3. Hayashi K, Hayashi H, Nakao F, Hayashi F. Risk factors for corneal endothelial injury during phacoemulsification. J Cataract Refract Surg. 1996;22(8):1079-1084.

4. Han JK, Miller KM. Heat production: longitudinal versus torsional phacoemulsification. J Cataract Refract Surg. 2009;35(10):1799-1805.

5. El-Moatassem Kotb AM, Gamil MM. Torsional mode phacoemulsification: effective, safe cataract surgery technique of the future. Middle East Afr J Ophthalmol. 2010;17(1):69-73.

6. Tognetto D, Cecchini P, Leon P, Di Nicola M, Ravalico G. Stroke dynamics and frequency of 3 phacoemulsification machines. J Cataract Refract Surg. 2012;38(2):333-342.

7. Christakis $\mathrm{P}$, Braga-Mele RM. Intraoperative performance and postoperative outcome comparison of longitudinal, torsional, and transversal phacoemulsification machines. J Cataract Refract Surg. 2012;38(2): 234-241.

8. Packer M, Fishkind WJ, Fine IH, Seibel BS, Hoffman RS. The physics of phaco: a review. J Cataract Refract Surg. 2005;31(2):424-431.

9. Liu Y, Zeng M, Liu X, et al. Torsional mode versus conventional ultrasound mode phacoemulsification: randomized comparative clinical study. J Cataract Refract Surg. 2007;33(2):287-292.

10. Fishkind W, Bakewell B, Donnenfeld E, Rose AD, Watkins LA, Olson RJ. Comparative clinical trial of ultrasound phacoemulsification with and without the WhiteStar system. J Cataract Refract Surg. 2006;32(1): 45-49.

11. Schmutz JS, Olson RJ. Thermal comparison of Infiniti OZil and Signature Ellips phacoemulsification systems. Am J Ophthalmol. 2010;149(5): 762e-767.e1.
Clinical Ophthalmology

\section{Publish your work in this journal}

Clinical Ophthalmology is an international, peer-reviewed journal covering all subspecialties within ophthalmology. Key topics include: Optometry; Visual science; Pharmacology and drug therapy in eye diseases; Basic Sciences; Primary and Secondary eye care; Patient Safety and Quality of Care Improvements. This journal is indexed on

\section{Dovepress}

PubMed Central and CAS, and is the official journal of The Society of Clinical Ophthalmology (SCO). The manuscript management system is completely online and includes a very quick and fair peer-review system, which is all easy to use. Visit http://www.dovepress.com/ testimonials.php to read real quotes from published authors. 